\title{
Thermodynamics, strange quark matter, and strange stars
}

\author{
G. X. Peng, ${ }^{1,2,3}$ H. C. Chiang, ${ }^{2}$ P. Z. Ning, ${ }^{3}$ \\ ${ }^{1}$ China Center of Advanced Science and Technology (World Laboratory), Beijing 100080, China \\ ${ }^{2}$ Institute of High Energy Physics, Chinese Academy of Sciences, Beijing 100039, China \\ ${ }^{3}$ Department of Physics, Nankai University, Tianjin 300071, China
}

\begin{abstract}
Because of the mass density-dependence, an extra term should be added to the expression of pressure. However, it should not appear in that of energy according to both the general ensemble theory and basic thermodynamic principle. We give a detail derivation of the thermodynamics with density-dependent particle masses. With our recently determined quark mass scaling, we study strange quark matter in this new thermodynamic treatment, which still indicates a possible absolute stability as previously found. However, the density behavior of the sound velocity is opposite to the previous finding, but consistent with one of our recent publication. We have also studied the structure of strange stars using the obtained equation of state.
\end{abstract}

PACS numbers: 12.39.-x, 24.85.+p, 05.70.-a, 97.10-q

\section{INTRODUCTION}

During the 10-plus years which have elapsed since Witten's conjecture [1] that strange quark matter (SQM), rather than the normal nuclear matter, might be the true ground state of quantum chromodynamics (QCD), many theoretical and observational efforts have been made on the investigation of its properties and potential astrophysical significance [2]. Because of the wellknown difficulty of QCD in the nonperturbative domain, phenomenological models reflecting the characteristic of strong interactions are widely used in the study of hadron, and many of them have been successfully applied to investigating the stability and properties of SQM. One of the most famous models is the MIT bag model with which Jaffe et. al [3] find that SQM is absolutely stable around the normal nuclear density for a wide range of parameters. A vast number of further investigations [4.5.6.7] are performed with fruitful results. A recent important result is that young millisecond pulsars are most likely to be strange stars rather than neutron stars 8 . Another alternative model is the mass-density-dependent model with which Chakrabarty et al. obtained significantly different results [9, 10]. However, Benvenuto and Lugones [11 pointed out that it is caused by the wrong thermodynamic treatment. They added an extra term to the expression of both pressure and energy, and got similar results as that in the bag model. A recent investigation indicates a link of SQM to the study of quark condensates [12] while a more recent work has carefully studied the relation between the charge and critical density of SQM [13.

Latterly, we have demonstrated that the previous treatments have unreasonable vacuum limits [14]. In addition to this problem, there exist another serious problem, i.e., the zero pressure does not appear in the lowest energy state. In fact, there are two important problems in the quark mass-density-dependent model. One is how to determine the quark mass scaling. The other is how to deal with the thermodynamics with density-dependent particle masses self consistently. We have mainly concentrated on the first problem in Ref. [14]. The present paper will concentrate more on the second problem. We find that the extra term provided in Ref. [11] should indeed be appended to the expression of pressure. However, it should not appear in that of energy according to both the general ensemble theory and basic thermodynamic principle. After our modification, the zero pressure point appears exactly at the lowest energy state, and thus the thermodynamics with density-dependent particle masses becomes self-consistent, which leads to completely different density behaviour of the sound velocity in SQM and different structure of strange stars.

We organize this paper as such. In the subsequent section, we give detail arguments on why the additional term in the pressure should not appear in the energy. The thermodynamic expression needed later are all derived carefully in this section. Then in Sec. III, we apply the new thermodynamic formulas to investigating strange quark matter, and find that the density behaviour of the sound velocity is opposite to the previous treatment but consistent with our recent publication. On application of our obtained equation of state, we integrate the equations of steller structure for strange stars in Sec. IV, and find that strange stars are dimensionally smaller and less massive than the previous calculation if SQM is absolutely stable. Sec. $\mathrm{V}$ is a short summary.

\section{THERMODYNAMICS WITH DENSITY-DEPENDENT PARTICLE MASSES}

Let us explore directly from the general ensemble theory what the expression of pressure and energy should look like if the particle masses are dependent on density.

We express the density matrix as

$$
\rho=\frac{1}{\Xi} e^{-\beta\left(E_{N_{i}, \alpha}-\sum_{i} \mu_{i} N_{i}\right)},
$$


where $\Xi$ is the partition function, $\beta$ is the reverse temperature, $N_{i}$ are the particle numbers, and $\mu_{i}$ are the corresponding chemical potentials. The microscopic energy $E_{N_{i}, \alpha}$ is a function of the system volume $V$, the particle masses $m_{i}$, the particle numbers $N_{i}$, and the other quantum numbers $\alpha$. The pressure of the system is

$$
\begin{aligned}
P & =\frac{1}{\Xi} \sum_{\left\{N_{i}\right\}, \alpha}\left(-\frac{\partial E_{N_{i}, \alpha}}{\partial V}\right) e^{-\beta\left(E_{N_{i}, \alpha}-\sum_{i} \mu_{i} N_{i}\right)} \\
& =\frac{1}{\Xi} \sum_{\left\{N_{i}\right\}, \alpha}\left[\frac{1}{\beta} \frac{\partial}{\partial V} e^{-\beta\left(E_{N_{i}, \alpha}-\sum_{i} \mu_{i} N_{i}\right)}\right] \\
& =\left.\frac{1}{\beta} \frac{\partial \ln \Xi}{\partial V}\right|_{T,\left\{\mu_{i}\right\}}=-\left.\frac{\partial(V \Omega)}{\partial V}\right|_{T,\left\{\mu_{i}\right\}},
\end{aligned}
$$

where

$$
\Omega \equiv-\frac{1}{V \beta} \ln \Xi
$$

is the thermodynamic potential density which is generally a function of the temperature $T$, the chemical potentials $\mu_{i}$, and the particle masses $m_{i}$. If the particle masses have nothing to do with the baryon number density $n_{b}=$ $N /(3 V)$ ( $\mathrm{N}$ is the total particle number), we simply get

$$
P=-\Omega
$$

If the masses depend on density or volume, one should have

$$
P=-\Omega+n_{b} \frac{\partial \Omega}{\partial n_{b}}
$$

This is just the right thing the authors have done in Ref. [11]. The authors derive it as such:

$$
P=-\left.\frac{\partial\left(\Omega / n_{b}\right)}{\partial\left(1 / n_{b}\right)}\right|_{T, \mu_{i}}=n_{b} \frac{\partial \Omega}{\partial n_{b}}-\Omega
$$

For canonical ensemble, the particle numbers $N_{i}$ keep fixed. This derivation is thus obvious. However, it is not so obvious for grand canonical ensemble because the particle number is not necessarily constant when the temperature $\mathrm{T}$ and chemical potentials $\mu_{i}$ unchanged. We will give a more convincing derivation a little later.

The additional term is of crucial importance for pressure balance. Not as done in Ref. [1], however, the extra term does not appear in the expression of energy. Now, let's calculate the statistic average for the energy:

$$
\begin{aligned}
\bar{E} & =\frac{1}{\Xi} \sum_{\left\{N_{i}\right\}, \alpha} E_{N_{i}, \alpha} e^{-\beta\left(E_{N_{i}, \alpha}-\sum_{i} \mu_{i} N_{i}\right)} \\
& =\frac{1}{\Xi} \sum_{\left\{N_{i}\right\}, \alpha}\left(-\frac{\partial}{\partial \beta}+\sum_{i} \mu_{i} N_{i}\right) e^{-\beta\left(E_{N_{i}, \alpha}-\sum_{i} \mu_{i} N_{i}\right)} \\
& =-\frac{\partial}{\partial \beta} \ln \Xi+\sum_{i} \mu_{i} \bar{N}_{i}
\end{aligned}
$$

where

$$
\begin{aligned}
\bar{N}_{i} & =\frac{1}{\Xi} \sum_{\left\{N_{i}\right\}, \alpha} N_{i} e^{-\beta\left(E_{N_{i}, \alpha}-\sum_{i} \mu_{i} N_{i}\right)} \\
& =\frac{1}{\Xi} \sum_{\left\{N_{i}\right\}, \alpha}\left[\frac{1}{\beta} \frac{\partial}{\partial \mu_{i}} e^{-\beta\left(E_{N_{i}, \alpha}-\sum_{i} \mu_{i} N_{i}\right)}\right]_{V, T,\left\{m_{k}\right\}} \\
& =\frac{1}{\beta} \frac{\partial}{\partial \mu_{i}} \ln \Xi=-\left.V \frac{\partial \Omega}{\partial \mu_{i}}\right|_{T,\left\{m_{k}\right\}}
\end{aligned}
$$

is the average number for particle type $i$. Therefore, the energy density of the system is

$$
\begin{aligned}
E & =\frac{\bar{E}}{V}=\frac{\partial(\beta \Omega)}{\partial \beta}+\sum_{i} \mu_{i} n_{i} \\
& =\Omega+\beta \frac{\partial \Omega}{\partial \beta}+\sum_{i} \mu_{i} n_{i} \\
& =\Omega+\sum_{i} \mu_{i} n_{i}-T \frac{\partial \Omega}{\partial T}
\end{aligned}
$$

where $n_{i}$ is the number density of particle type $i$ :

$$
n_{i} \equiv \frac{\bar{N}_{i}}{V}=-\left.\frac{\partial \Omega}{\partial \mu_{i}}\right|_{T,\left\{m_{k}\right\}} .
$$

It is clear from Eq. (11) that only when Eq. (4) holds can one get the Eq. (8) in Ref. [11]. Therefore, we should not, as done in Ref. [11], use that expression to calculate the energy density. Instead, we will calculate $E$ directly from Eq. (11) in this paper.

For more evident arguments, let's see the following derivation starting from the basic derivative relation for an open system:

$$
d(V E)=T d(V S)-P d V+\sum_{i} \mu_{i} d \bar{N}_{i}
$$

where $\mathrm{S}$ is the entropy density of the system.

Choosing T, V, and $\left\{\bar{N}_{i}\right\}$ as the independent macroscopic state variables, the combined statement of the first and second laws of thermodynamics, Eq. (13), can be expressed as

$$
d(V A)=-V S d T-P d V+\sum_{i} \mu_{i} d \bar{N}_{i}
$$

where $A \equiv E-T S$ is the Helmholtz free energy density by which we have

$$
\begin{aligned}
P & =-\left.\frac{d(V A)}{d V}\right|_{T,\left\{\bar{N}_{i}\right\}} \\
& =-A-\left.V \frac{d A}{d V}\right|_{T,\left\{\bar{N}_{i}\right\}} \\
& =-A+\left.\sum_{j} n_{j} \frac{d A\left(T,\left\{n_{i}\right\}\right)}{d n_{j}}\right|_{T} .
\end{aligned}
$$


This is a general expression for pressure. In obtaining the third equality, we have used the chain relation

$$
-\left.V \frac{d}{d V} f\left(\left\{n_{i}=\frac{\bar{N}_{i}}{V}\right\}\right)\right|_{\left\{\bar{N}_{i}\right\}}=\sum_{j} n_{j} \frac{d}{d n_{j}} f\left(\left\{n_{i}\right\}\right),
$$

where $f$ is an arbitrary function.

According to the basic relation between thermodynamics and statistics, we have

$$
A=\Omega+\sum_{i} \mu_{i} n_{i}
$$

where $\Omega$ is the thermodynamic potential density. For a free Fermi system, it is

$$
\begin{aligned}
\Omega & =-\sum_{i} \frac{g_{i} T}{2 \pi^{2}} \int_{0}^{\infty} \ln \left[1+e^{-\beta\left(\sqrt{p^{2}+m_{i}^{2}}-\mu_{i}\right)}\right] p^{2} d p \\
& \equiv \sum_{i} \Omega_{i}\left(T, \mu_{i}, m_{i}\right)
\end{aligned}
$$

where $g_{i}$ is the degeneracy factor which is 6 for quarks and 2 for electrons. In order to include the interaction between particles, we regard the particle masses as densitydependent, namely

$$
m_{i}=m_{i}\left(n_{b} \equiv \sum_{j} n_{j} / 3\right)
$$

Because we have chosen $T, V$, and $\left\{\bar{N}_{i}\right\}$ as independent state variables, the chemical potential $\mu_{i}$ should also be regarded as a function of $\mathrm{T}$ and $\left\{n_{k}\right\}$, namely

$$
\mu_{i}=\mu_{i}\left(T,\left\{n_{k}\right\}\right)
$$

So, the total derivative of $\Omega\left(T,\left\{\mu_{k}\right\},\left\{m_{k}\right\}\right)$ with respect to $n_{j}$ should be taken like this:

$$
\begin{aligned}
\left.\frac{d \Omega}{d n_{j}}\right|_{T} & =\left.\left.\sum_{i} \frac{\partial \Omega}{\partial \mu_{i}}\right|_{T,\left\{m_{k}\right\}} \frac{d \mu_{i}}{d n_{j}}\right|_{T}+\left.\frac{\partial \Omega}{\partial n_{b}}\right|_{T,\left\{\mu_{k}\right\}} \frac{\partial n_{b}}{\partial n_{j}} \\
& =-\left.\sum_{i} n_{i} \frac{d \mu_{i}}{d n_{j}}\right|_{T}+\left.\frac{1}{3} \frac{\partial \Omega}{\partial n_{b}}\right|_{T,\left\{\mu_{k}\right\}} .
\end{aligned}
$$

Here we have used the Eq. (12) and the fact that $\partial n_{b} / \partial n_{j}=1 / 3$.

Substituting Eq. (19) into Eq. (17) gives

$$
\begin{aligned}
P & =-A+\sum_{j} n_{j} \frac{d}{d n_{j}}\left[\Omega+\sum_{i} \mu_{i} n_{i}\right]_{T} \\
& =-A+\sum_{j} n_{j}\left[\left.\frac{d \Omega}{d n_{j}}\right|_{T}+\sum_{i}\left(\left.n_{i} \frac{d \mu_{i}}{d n_{j}}\right|_{T}+\mu_{i} \frac{d n_{i}}{d n_{j}}\right)\right] \\
& =-A+\sum_{i} \mu_{i} n_{i}+\left.\sum_{j} \frac{n_{j}}{3} \frac{\partial \Omega}{\partial n_{b}}\right|_{T,\left\{\mu_{k}\right\}} \\
& =-\Omega+\left.n_{b} \frac{\partial \Omega}{\partial n_{b}}\right|_{T,\left\{\mu_{k}\right\}} \\
& =\sum_{i}\left(-\Omega_{i}+n_{b} \frac{\partial m_{i}}{\partial n_{b}} \frac{\partial \Omega_{i}}{\partial m_{i}}\right)
\end{aligned}
$$

According to the Pauli principle and the relativistic energy-momentum relation $\varepsilon_{i}=\sqrt{p^{2}+m_{i}^{2}}$, the energy (28)density of the system at zero temperature should be

$$
E\left(\left\{n_{i}\right\},\left\{m_{j}\left(n_{b}\right)\right\}\right)=\sum_{i} \frac{g_{i}}{2 \pi^{2}} \int_{0}^{p_{f, i}} \varepsilon_{i} p^{2} d p
$$

which, when the integration is carried out, is just the same as Eq. (33). 
Because the entropy is also zero (or constant) at zero temperature, we can substitute Eq. (41) into Eq. (40), and accordingly get

$$
\begin{aligned}
P & =-E+\sum_{j} n_{j}\left(\frac{\partial E}{\partial n_{j}}+\sum_{i} \frac{\partial E}{\partial m_{i}} \frac{\partial m_{i}}{\partial n_{b}} \frac{\partial n_{b}}{\partial n_{j}}\right) \\
& =-E+\sum_{j} n_{j} \frac{\partial E}{\partial n_{j}}+\sum_{i} \sum_{j} \frac{n_{j}}{3} \frac{\partial E}{\partial m_{i}} \frac{\partial m_{i}}{\partial n_{b}} \\
& =-\Omega+\sum_{i} n_{b} \frac{\partial m_{i}}{\partial n_{b}} \frac{\partial E}{\partial m_{i}},
\end{aligned}
$$

which leads to Eq. (34) exactly.

\section{PROPERTIES OF STRANGE QUARK MATTER IN THE NEW THERMODYNAMIC TREATMENT}

Having derived in detail the thermodynamics with variable particle masses in the previous section, we now apply it to the investigation of strange quark matter.

As usually done in the previous literature [3, 9, 19, 11, 12, 13, 14, We assume the SQM to be a Fermi gas mixture of $u, d, s$ quarks and electrons with chemical equilibrium maintained by the weak interactions:

$$
d, s \leftrightarrow u+e+\bar{\nu}_{e}, s+u \leftrightarrow u+d, \ldots
$$

Because of these reactions, the chemical potentials $\mu_{i}(i=u, d, s, e)$ should satisfy

$$
\begin{aligned}
& \mu_{d}=\mu_{s} \equiv \mu, \\
& \mu_{u}+\mu_{e}=\mu .
\end{aligned}
$$

For the bulk SQM in weak equilibrium, the previous investigations got a slightly positive charge [3]. Our recent study [13] demonstrates that negative charges could lower the critical density. However, too much negative charge can make it impossible to maintain flavor equilibrium. Therefore, the charge of SQM is not allowed to shift too far away from zero at both positive and negative directions. Therefore, one also has another two equations for a given baryon number density $n_{b}$ :

$$
\begin{gathered}
\frac{1}{3}\left(n_{u}+n_{d}+n_{s}\right)=n_{b}, \\
\frac{2}{3} n_{u}-\frac{1}{3} n_{d}-\frac{1}{3} n_{s}-n_{e}=0 .
\end{gathered}
$$

The first is the definition of baryon number density; the second is from the charge neutrality requirement. $n_{i}(i=$ $u, d, s, e)$ is related to $\mu_{i}$ and $m_{i}$ by Eq. (32).

Because the results from lattice calculations show that quark matter does not become asymptotically free soon after the phase transition (instead, it approaches the free gas equation of state very slowly), one should consider the strong interaction between quarks in a proper way.
We do this by including the interaction effect within the variable quark masses. Because of the characteristic of the quark confinement and asymptotic freedom of the strong interaction, one can write down the simplest and most symmetric parametrization for the quark masses $m_{q}(q=u, d, s)$ [14]:

$$
m_{q}=m_{q 0}+\frac{D}{n_{b}^{z}}
$$

where $m_{q 0}$ is the corresponding quark current mass, $z$ is a fixed exponential. Previously, $z$ is regarded as 1 . Our recent study [14] indicates that it is more reasonable to take $z=1 / 3$. The parameter $D$ is usually determined by stability arguments, i.e., at zero pressure $(P=0)$, the energy per baryon, $E / n_{b}$, is great than $930 \mathrm{MeV}$ for two flavor quark matter in order not to contradict standard nuclear physics, but less than $930 \mathrm{MeV}$ for three flavor symmetric quark matter so that SQM can have the possibility of absolute stability. Obviously, the rang of $D$ determined by this method depends on different thermodynamic treatments. Within the thermodynamics derived in the preceding section, $D$ is in the range (155$171 \mathrm{MeV})^{2}$ when taking $z=1 / 3$.

Because the light quark current masses are very small, their value uncertainties are not important. So we take the fixed central values $m_{u 0}=5 \mathrm{MeV}$ and $m_{d 0}=10$ $\mathrm{MeV}$ in our calculation. The electron mass is very small $(0.511 \mathrm{MeV})$. As for $s$ quarks, we take 80 and $90 \mathrm{MeV}$, corresponding respectively to $D^{1 / 2}=156$ and $160 \mathrm{MeV}$.

For a given $n_{b}$, we solve for $\mu_{i}(i=u, d, s, e)$ from Eqs. (45) - (48), and calculate the energy density and pressure of SQM respectively from Eqs. (33) and (34) with the quark messes replace by Eq. (49).

Firstly, we draw the configuration of the SQM for the parameter set $m_{s 0}=80 \mathrm{MeV}$ and $D^{1 / 2}=156 \mathrm{MeV}$ in Fig. 1. At high densities, all of the $u, d$, and $s$ quarks tend to become a triplicate. When the density becomes lower, $d$ fraction will increases while $s$ fraction decreases, and will become zero at a definite density which is called critical density in Ref. [13] because SQM does not exist below that density. The $u$ fraction is nearly unchanged. It in fact increases very slowly. To keep charge neutrality, the electron fraction will also increase. However, because of its very small mass, the electron fraction is so little that we multiply by one thousand to draw it in the figure.

In Fig. 2, we show the density dependence of the energy per baryon, $V E / N=E / n_{b}$, vs baryon number density $n_{b}$ for the parameter set I: $m_{s 0}=80 \mathrm{MeV}, D^{1 / 2}=156$ $\mathrm{MeV}$, and II: $m_{s 0}=90 \mathrm{MeV}, D^{1 / 2}=160 \mathrm{MeV}$. For the first parameter set, SQM is absolutely stable while for the second set it is nearly meta-stable. The point marked with a circle ' $\mathrm{O}$ ' is the zero pressure point where the system pressure becomes zero. It can be clearly seen that the zero pressure points are exactly located at the lowest energy state. In fact, this is a basic requirement of thermodynamics because one can obtain from Eq. (39) 


$$
P=-\left.\frac{d(V E)}{d n_{b}} \frac{d n_{b}}{d V}\right|_{\left\{\bar{N}_{k}\right\}}=-n_{b}^{2} \frac{d\left(E / n_{b}\right)}{d n_{b}} .
$$

However, this is not the case for most of the previous thermodynamic treatments of strange quark matter in the mass-density-dependent model [11, 9, 10, 12, which is their another serious flaw in addition to the unreasonable vacuum limits mentioned before.

In Fig. 3, we give the relation between the pressure $P$ and energy density $E$, i.e., the equation of state. It approaches the free gas equation of state at high densities. However, its shape is a little sunken at lower densities, contrary to previous calculation which is protuberant. This will leads to completely different lower density behaviour of the sound velocity in strange quark matter.

The velocity of sound is plotted in the lower part of Fig. $\$$. The upper part is calculated by the same method in Ref. 11] with parameter set B there. Simultaneously given with a full horizontal line is the ultra-relativistic case $(1 / \sqrt{3})$ for purpose of comparison. Obviously, they become nearly identical at high densities while the lower density behaviour is opposite. The sound velocity in the previous treatment is higher than the ultra-relativistic case and will eventually exceed the speed of light at lower densities, which is unreasonable from the point of view of the theory of relativity.

\section{STRANGE STARS}

It has long been proposed that the currently called neutron stars might be composed of strange quark matter and thus be in fact strange stars. A recent investigation shows that young millisecond pulsars are most likely to be strange stars rather than neutron stars [8]. Previous authors have investigated the properties of strange stars by applying their obtained equation of state with interesting results [10,11]. We have now modified the thermodynamic treatment and updated the quark mass scaling. Therefore, it is meaningful to study the structure of strange stars in the new context from the astrophysical view point.

As generally done, we assume the strange star to be a spherically symmetric object. Its stability is determined by the general relativistic equation of hydrostatic equilibrium known as Tolman-Oppenheimer-Volkov equation [16]

$$
\frac{d P}{d r}=-\frac{G m E}{r^{2}} \frac{\left(1+4 \pi r^{3} P / m\right)(1+P / E)}{1-2 G m / r},
$$

with the subsidiary condition

$$
d m / d r=4 \pi r^{2} E,
$$

where $G=6.707 * 10^{-45} \mathrm{MeV}^{-2}$ is the gravitational constant, $r$ is the distance from the core of the star, $E=E(r)$ is the energy density or mass density, $P=P(r)$ is the pressure, and $m=m(r)$ is the mass within the radius $r$.

For an initial baryon number density $n_{0}$ (accordingly $P_{0}$ and $E_{0}$ ), we can numerically solve Eqs. (51) and (52) with the aid of the equation of state, and obtain the corresponding $P=P\left(r, n_{0}\right)$ and $m=m\left(r, n_{0}\right)$, and consequently $n=n\left(r, n_{0}\right)$, the baryon number density at the radius $r$ for the central density $n_{0}$. The radius $R$ of the strange star is determined by the condition

$$
P\left(R, n_{0}\right)=0,
$$

namely,

$$
R=R\left(n_{0}\right) .
$$

Accordingly, the mass of the strange star is

$$
M=m\left(R\left(n_{0}\right), n_{0}\right) \equiv M\left(n_{0}\right) .
$$

To make strange stars stable, we must require $d M / d n_{0}>0$. For the above obtained equation of state, M firstly increases with $n_{0}$ up to a definite value $M_{\max }$ corresponding to the highest acceptable central density $n_{0 \max }$. After that, $M$ decreases with $n_{0}$, and the star becomes unstable.

For the parameter set I, i.e. $m_{s 0}=80 \mathrm{MeV}$ and $D=(156 \mathrm{MeV})^{2}$, we give the density profiles $n\left(r, n_{0}\right)$ in Fig. 5 as an example. The upmost line is for the largest acceptable central density $n_{0 \max }\left(\approx 1.35 \mathrm{fm}^{-3}\right)$. The lowest horizontal line corresponds to the surface density $n_{s}\left(\approx 0.25 \mathrm{fm}^{-3}\right)$ of strange stars which is independent of the central density, but a functional of the equation of state. Each line will intersect with it. The cross points correspond to the radius $R$ of the star. The maximum radius of the star appears in $n_{0} \approx 0.65 \mathrm{fm}^{-3}$.

In Fig. 6, we show the mass-radius relation of strange stars with a solid line. The point marked with a full dot ' $\bullet$ ' represents the largest acceptable mass $M_{\max }(\approx$ 1.58 times the solar mass). For comparison, we have also plotted the result from the bag model calculation with the bag constant $B^{1 / 4}=144 \mathrm{MeV}$, and that in Re. 11] with the parameter set B there. We can see that the strange stars in our case is dimensionally smaller and less massive than the previous calculation if SQM is absolutely stable. Naturally, this observation depends on the parameters employed. If we choose a bigger $m_{s 0}$ and larger $D$, the case would be different. However, SQM would have no possibility of absolute stability in that case.

\section{SUMMARY}

We have derived the thermodynamics with densitydependent particle masses self-consistently, which overcome the serious flaws of the previous treatment of SQM in the quark mass-density-dependent model. We find 
that an additional term should be appended to the expression of pressure, but it should not appear in that of energy. When applying the new formulas to the investigation of SQM, we find that the density behaviour of the sound velocity is opposite to the previous calculation, but consistent with our recent publication, which leads to different structure of strange stars.

\section{ACKNOWLEDGMENTS}

The authors would like to thank the National Natural Science Foundation of China for financial support under Grant No. 19905011.

[1] E. Witten, Phys. Rev. D 30, 272 (1984).

[2] L.M. Zhou, G.X. Peng, and P.Z. Ning, Prog. Phys. 19, 59 (1999).

[3] E. Farhi and R.L. Jaffe, Phys. Rev. D 30, 2379 (1984); M.S. Berger and R. L. Jaffe, Phys. Rev. C 35, 213 (1987); E.P. Gilson and R. L. Jaffe, Phys. Rev. Lett. 71, 332 (1993).

[4] Jes Madsen, Phys. Rev. Lett. 61, 2909 (1993); Phys. Rev. D 47, 5156 (1993); 50, 3328 (1994); Jes Madsen, Dan M. Jensen, and Michael B. Christiansen, Phys. Rev. C 53, 1883 (1996).

[5] B.C. Parija, Phys. Rev. C 48, 2483 (1993); 51, 1473 (1995).

[6] L. Satpathy, P.K. Sahu, and V.S. Uma Maheswari, Phys. Rev. D 49, 4642 (1994).

[7] Jürgen Schaffner-Bielich, Carsten Greiner, Alexander Diener, and Horst Stöcker, Phys. Rev. D 55, 3038 (1997).

[8] J. Madsen, Phys. Rev. Lett. 81, 3311 (1998).

[9] S. Chakrabarty, S. Raha, and B. Sinha, Phys. Lett. B 229, 112 (1989).

[10] S. Chakrabaty, Phys. Rev. D 43, 627 (1991); 48, 1409 (1993); 54, 1306 (1996).

[11] O.G. Benvenuto and G. Lugones, Phys. Rev. D 51, 1989 (1995); G. Lugones and O.G. Benvenuto, ibid. 52, 1276 (1995).

[12] G.X. Peng, P.Z. Ning, and H.C. Chiang, Phys. Rev. C 56, 491 (1997).

[13] G.X. Peng, H.C. Chiang, P.Z. Ning, and B.S. Zou, Phys. Rev. C 59, 3452 (1999).

[14] G.X. Peng, H.C. Chiang, J.J. Yang, L. Li, and B. Liu, Phys. Rev. C 61, 015201 (2000).

[15] A. Ukawa, Nucl. Phys. A 498, 227c (1989). V.M. Belyaev and Ya.I. Kogan, Phys. Lett. B 136, 273 (1984); K.D. Born, E. Laermann, N. Pirch, T.F. Walsh, and P.M. Zerwas, Phys. Rev. D 40, 1653 (1989).

[16] V. M. Lipunov, Astrophysics of Neutron Stars, translated into English version by R. S. Wadhwa, Springer-Verlag, 1992.
FIG. 1. The configuration of SQM varies with density. At high densities, all of the $u, d$, and $s$ quarks tends to become a triplicate. When the density becomes lower, $d$ fraction will increases while the $s$ fraction decreases. The $u$ fraction is nearly unchanged. It only increases very slowly.

FIG. 2. Energy per baryon $E / n_{b}$ vs baryon number density $n_{b}$ for differnt parameter groups. The zero pressure points marked with ' $O$ ' are located at the lowest energy state, which is not the case for most of the previous thermodynamic treatments of strange quark matter in the quark mass-density-dependent model.

FIG. 3. Equation of state of strange quark matter (pressure $\mathrm{P}$ vs energy density $\mathrm{E}$ ). it approaches the free gas equation of state at high densities. However, it is a little sunken at lower densities, contrary to previous calculation.

FIG. 4. Velocity of sound in strange quark matter. The solid horizontal line is the ultra-relativistic case. the lower half part is the results of our calculation while the upper part is calculated by the same method in Re. 11 for parameter set B there. Their lower density behaviour is obviously opposite.

FIG. 5. Density profiles for parameter set $m_{s 0}=80 \mathrm{MeV}$ and $D=(156 \mathrm{MeV})^{2}$. The upmost line is for the largest acceptable central density $n_{0 \max }$. The lowest horizontal line corresponds to the surface density of strange stars. The cross points of each line and the lowest horizontal line correspond to the radius $R$ of the star.

FIG. 6. Mass-Radius relation for strange stars. The vertical axis is the star mass in unit of the solar mass while the horizontal axis is the star radius in unit of kilometer. The solid line is obtained by the method in this paper. The dotted line is from the bag model. The dashed line is calculated with the same method in Ref. [11] for the parameter set B there. The points marked with a full dot ' $\bullet$ ' represent the maximum acceptable masses. 


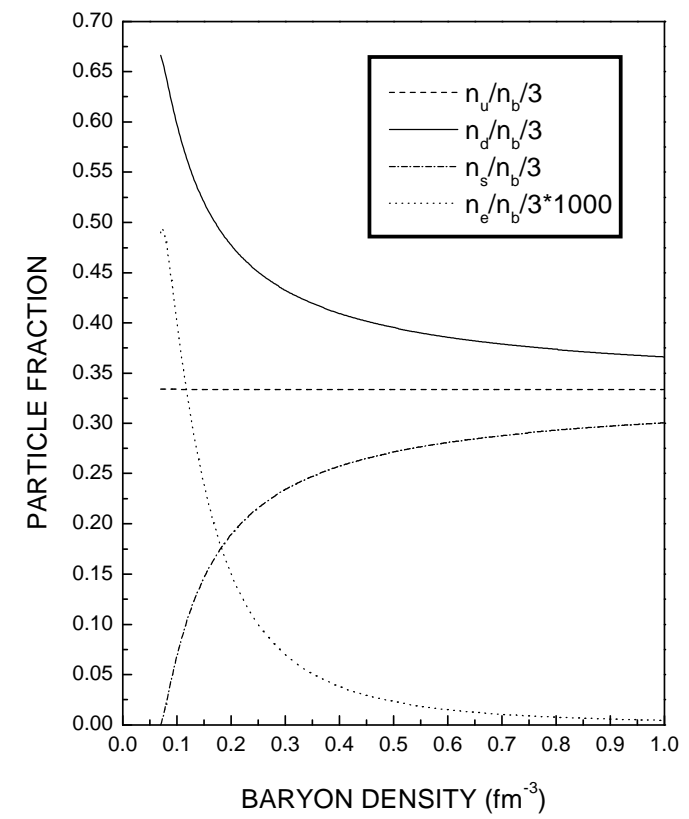




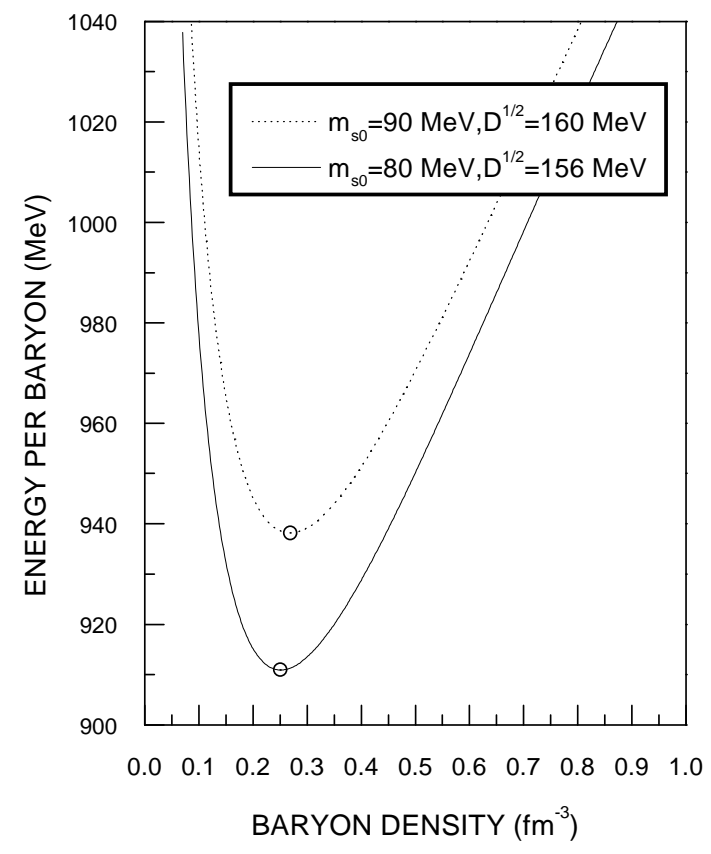




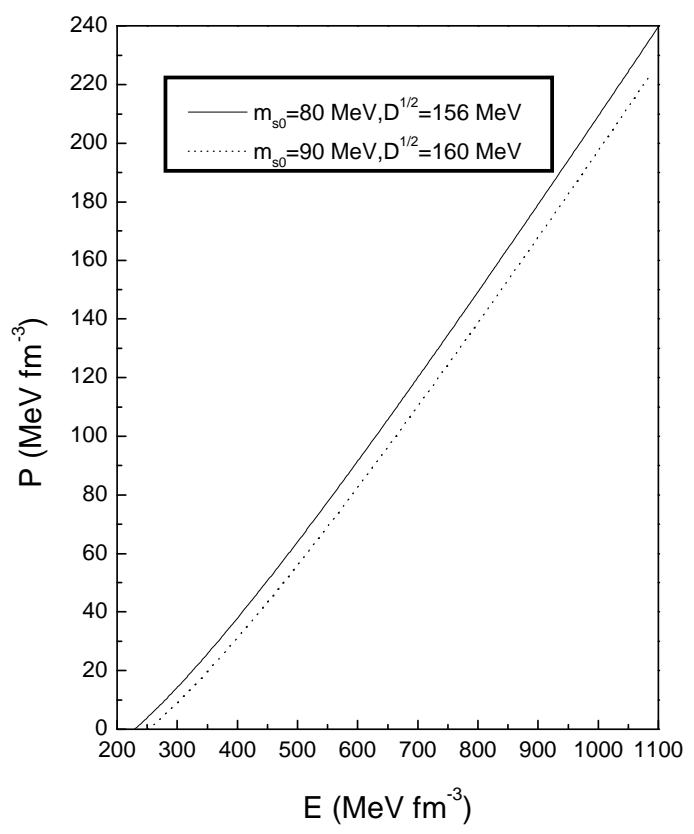




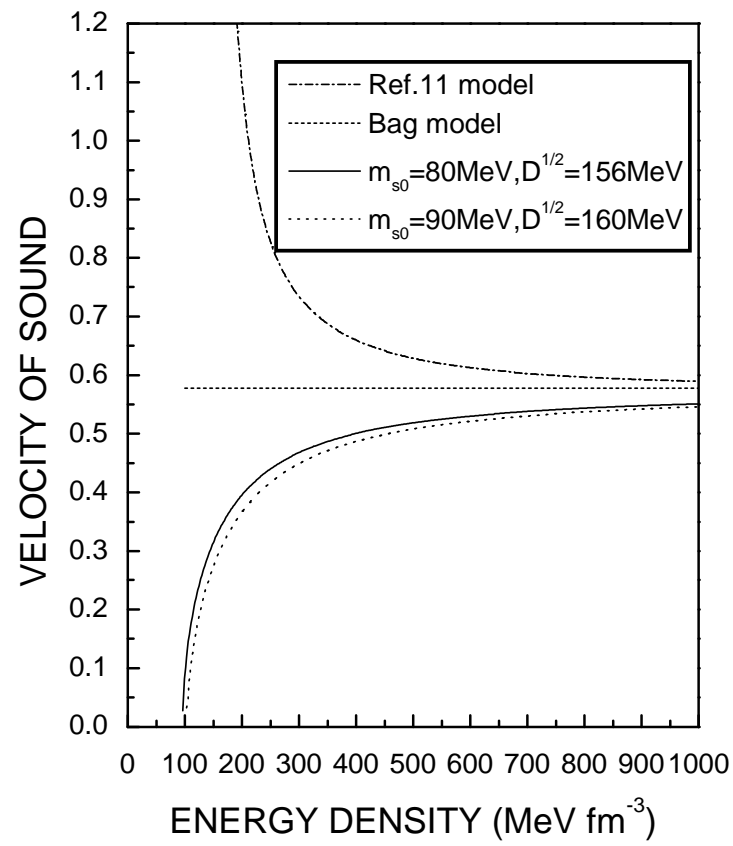




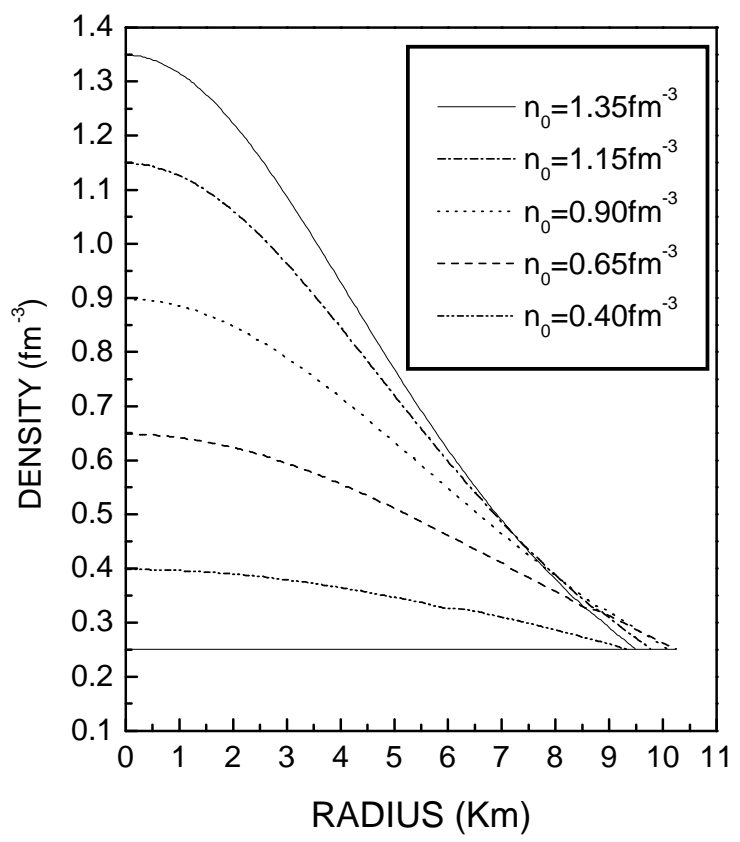




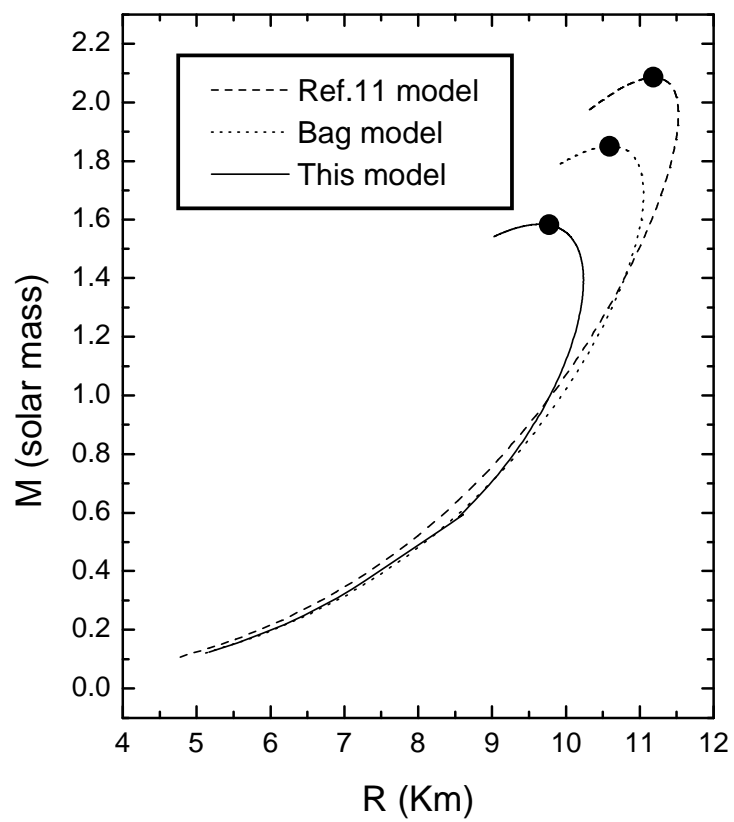

